\title{
Valoración económica de la demanda del servicio ambiental hídrico del bosque de la micro cuenca Madriguera, Siuna Nicaragua
}

\author{
Economic valuation of the demand for the environmental water service \\ of the forest of the Madriguera micro watershed, Siuna, Nicaragua
}

Oscar Flores Pérez ${ }^{1}$

\section{Resumen}

F ste estudio estimó la disponibilidad a pagar (DAP) de las familias residentes en el área urbana del - 1 municipio de Siuna, que reciben agua para consumo diario de la microcuenca Madriguera, por la mejora en la oferta hídrica, mediante el método de valoración contingente y el uso de formato binario. Se estimaron medidas de cambio en el bienestar a través de estimaciones paramétricas en la forma funcional lineal. Asimismo, se calcularon los componentes del valor económico total que los beneficiarios asignan al recurso hídrico proveniente del bosque de la microcuenca. Los resultados de la disposición a pagar (DAP), han revelado que las viviendas que tienen fuente hídrica alterna, tienen una menor disposición a pagar y viceversa. Una alta proporción de la población está dispuesta a pagar y lo hacen de acuerdo a los tipos de valor económico de uso directo y de herencia. Quienes no están dispuestos a pagar es porque no tienen recursos económicos. El valor mínimo como medida de cambio de bienestar es de $C \$ 116.29$ (Ciento dieciséis con 29córdobas). Ello implica la cantidad de $C \$ 74,890.76$ mensuales para un total de $C \$ 898,689.12$ anualmente. Los beneficiarios reconocen la importancia del bien y se justifica su valoración en cuanto a que el servicio ambiental de la microcuenca les genera bienestar y favorecen la idea de implementar proyectos de protección y conservación para evitar un cambio desfavorable.

Palabras clave: microcuenca; oferta hídrica; medidas de bienestar; disponibilidad a pagar; valoración contingente.

\section{Summary}

This study estimated the willingness to pay (DAP) of the families residing in the urban area of the municipality of Siuna, who receive water for daily consumption from the Madriguera micro-watershed, due to the improvement in the water supply, through the contingent valuation method and the use of binary format. Measures of change in welfare were estimated through parametric estimates in the linear functional form. Likewise, the components of the total economic value that the beneficiaries assign to the water resource from the forest of the micro-watershed were calculated. The results of the willingness to pay (DAP), have revealed that the houses that have alternative water source, have a lower willingness to pay and vice versa. A high proportion of the population is willing to pay and they do so according to the types of economic value of direct use and inheritance. Those who are not willing to pay is because they do not have economic resources. The minimum value as a measure of welfare change is $C \$ 116.29$ (One hundred and sixteen with 29 cents). This implies the amount of $C \$ 74,890.76$ monthly for a total of $C \$ 898,689.12$ annually. The beneficiaries recognize the importance of the good and their assessment is justified in that the environmental service of the micro-watershed generates well-being and favors the

\footnotetext{
Máster en Docencia Universitaria. Profesor de la Universidad de las Regiones Autónomas de la Costa Caribe Nicaragüense-Recinto Universitario Las Minas. Correo: oscar.flores@uraccan.edu.ni
} 
idea of implementing protection and conservation projects to avoid an unfavorable change.

Key Words: micro watershed; water supply; welfare measures; availability to pay; contingent valuation.

\section{Introducción}

Los impactos sociales, económicos y ambientales que se producen por manejo inadecuado de los bosques cada día son más significativos y, Villena y Lafuente (2012) en un ecosistema el valor de todo es mayor que la sumatoria de sus partes (p.1). De acuerdo con Martínez y Dimas la teoría económica establece que las externalidades negativas son fallas de mercado (2011, pág. 25) y, la no incorporación de los costos ambientales en los costos productivos obedece según Larach (2000) a la "existencia de fallas de políticas y de mercado, donde el precio del bien no refleja ni su valor ni su costo social marginal" (p. 17).

El concepto de valor económico, se basa en la idea utilitarista. "El medio ambiente, bajo esta perspectiva, tiene valor en cuanto que proporciona beneficios al ser humano" (Cerda, 2003, p.135). El estudio valoró económicamente la demanda del servicio ambiental hídrico, cuya parte agua está en el bosque de la microcuenca Madriguera, en las familias residentes en la municipalidad de Siuna, a través del método de Valoración Contingente (VC) con formato binario, a fin de conocer las posibles contribuciones (DAP) que podrían realizar los beneficiarios a favor de la protección y conservación de este bosque. Se planteó para ello, un escenario de valoración, donde se mejorará la calidad de este recurso a través de las gestiones a realizar en la microcuenca a fin de conseguir el servicio hídrico en sus hogares, de manera estable, en cantidad y calidad.

Para conocer las medidas de cambios en el bienestar se utilizaron estimaciones paramétricas bajo el modelo de diferencia de la función indirecta de utilidad, con distribuciones Probit y Logit para los errores $y$ las formas funcionales lineales.

\section{Revisión de la literatura}

\section{Método de valoración contingente (MVC)}

"La valoración contingente, es una de las metodologías más utilizadas para asignar un valor monetario a los recursos ambientales que el mercado no puede traducir directamente en precios" (Hanemann, 2012, pág. 31). Según Cerda, Rojas y García este método se basa en intenciones de conducta del consumidor en mercados hipotéticos (2007, p. 147). En este método, "los cuestionarios juegan el papel de un mercado hipotético, donde la oferta viene representada por la persona entrevistadora y la demanda por la entrevistada" (Riera, 1994, p 8).

Desde la perspectiva económica, el Método de Valoración Contingente (MVC) se basa en la maximización de la utilidad de los entrevistados, donde la función de utilidad $(U)$ de los individuos está definida para bienes de mercado y no mercado, configurados por cantidades, precios y otros atributos (Aviléz, y otros, 2010, p. 118). Otro componente es la función de utilidad indirecta, constituida por un elemento estocástico, que representa la aleatoriedad y constituye el modelo de maximización de la utilidad aleatoria.

Los individuos pueden optar por una mejora en la calidad del bosque, por la que deben pagar una cantidad $b t$, donde $t$ indica las distintas cantidades que se ofrecen a diferentes individuos (vector de pago) por obtener una mejora (Cerda, García, Bahamondez, \& Poblete, 2010, p. 200).

El Método de Valoración Contingente (MVC) se fundamenta en la teoría de la elección racional del consumidor, es decir, se supone que "los individuos realizan decisiones de consumo que maximizan su nivel de bienestar" (Salazar S. d., 2000, pág. 9).

Las estimaciones de los modelos de este estudio se realizaron con base en el modelo de Hanemann (1984), conocido como diferencia en la función de utilidad indirecta.

Las medidas de Variación Compensada (VC) o Variación Equivalente (VE) tienen carácter aleatorio y, puesto que, los usuarios no tienen derecho de propiedad sobre el bien a valorar se uso la variación equivalente, y esta medida de bienestar es capturada por una pregunta de disposición a pagar (DAP). Además, se optó por el formato dicotómico sugerido por Hanemann (1984) considerando que es técnicamente mejor, frente al enfoque de Cameron (1988), ya que resulta más explícito el papel que juegan los cambios de bienestar en el proceso de 
decisión del individuo (Cerda, Rojas, y García, 2007, p. 147)

\section{Medidas de bienestar}

La base del método de valoración contingente es estimar cambios en el bienestar de las personas cuando cambia la calidad de un bien ambiental (Barzev, 2002, p. 37)

"El bienestar de los individuos no solamente depende del consumo de bienes y servicios producidos por el sector privado y el gobierno, sino también de cantidades y calidades de flujos de bienes y servicios no mercantiles, provistos por el sistema de recursos naturales y ambientales" (Matínez \& Dimas, 2011, p. 46). Cuando un individuo maximiza su utilidad sujeta a su restricción presupuestaria, su función indirecta de utilidad es decreciente en los precios y creciente en la renta y en la calidad ambiental.

Si se introduce ahora un cambio en la calidad ambiental, por simplicidad, se asume que los precios y la renta no cambian. Entonces, el cambio en la utilidad es: $\Delta V=V\left(p, y, z^{1}\right)-V\left(p, y, z^{0}\right)$ donde el superíndice o (1) indica el valor inicial (final) de la calidad ambiental. Dado que la función de utilidad no es observable, se necesita una medida monetaria para valorar el cambio en la utilidad (Salazar., 2000, p. 67).

La variación compensatoria $(V C)$ es una cantidad de dinero tal que: $\left.V\left(p, y-V C, z^{1}\right)\right)=V(p, y$, $\left.z^{0}\right)$. En otras palabras, la VC es disposición a pagar (DAP) por una mejora en la calidad ambiental. No obstante, si la calidad ambiental se deteriora es la cantidad mínima que habría que darle al individuo para compensarle por la pérdida en la calidad ambiental, en este caso, la VC mide la disposición a ser compensado (DAC) por un deterioro en la calidad ambiental.

Por su parte, la variación equivalente (VE) es una cantidad de dinero tal que: $V\left(p, y+V E, z^{o}\right)$ $=V\left(p, y, z^{1}\right)$ puesto, que el escenario en estudio, enfatiza un potencial deterioro del bosque de la Microcuenca Madriguera, lo cual traería como consecuencia, disminución del servicio hídrico que genera el bosque, esto lo haría más escaso como bien de calidad, por tanto, sería mejor valorado, es decir, incrementaría su valor (precio). Basado en esta premisa, se eligió la variación equivalente $(V E)$, donde no se da el cambio, si no que con el proyecto se evitaría el cambio desfavorable y además es el caso donde la persona afectada (beneficiario distante) no tiene el derecho de propiedad.

El uso de una u otra modalidad depende en gran medida de la definición de los derechos de propiedad sobre el bien que se desea valorar.

La Variación Equivalente (V.E) función de gasto del individuo. Se traduce en la diferencia en el gasto necesario para alcanzar el nuevo nivel de bienestar, evitando un cambio desfavorable en el bien ambiental, dado un nivel de precios $(P)$ y el nivel de utilidad después de la implementación del programa de conservación (U1), (U0 es el nivel de utilidad antes de la intervención humana que deteriora la calidad ambiental) (Mendieta, 200o, p. 231), en este caso, si la calidad ambiental se deteriora. La variación equivalente (V.E) es la cantidad máxima que el individuo está dispuesto a pagar para prevenir dicho deterioro.

\section{Estructura de los modelos}

Kriström (1990), plantea un estimador no paramétrico apropiado para ejercicios de valoración contingente con formato de pregunta discreto o binario. Frente a las aproximaciones paramétricas más tradicionales presenta dos ventajas: no es necesario suponer una forma funcional para la distribución de la DAP y es relativamente claro y sencillo de calcular. Como señalan Pommerehne y Hart (1997), aquí lo importante es disponer de una muestra lo suficientemente grande y representativa de la población para poder obtener unos valores directos y fiables de la media y mediana de la DAP.

Este estimador está relacionado con la teoría de la utilidad a través de un argumento de primer orden, dado que las probabilidades de aceptar un pago dependerán solamente de la magnitud del precio de salida propuesto. Por lo tanto, los datos son agrupados de tal forma que para cada precio de salida tenemos la proporción de respuestas positivas. Se puede demostrar que la estimación por máxima verosimilitud de la probabilidad de una respuesta positiva es la proporción de respuestas positivas i para cada precio de salida Ai. Teniendo una media igual a $\pi_{i}$ y una varianza $\pi_{i}\left(1-\pi_{i}\right) n_{i}$, siendo ni el número de individuos del grupo i.

En el caso típico de un estudio de valoración contingente con variable dependiente discreta, donde se tienen diferentes precios de salida dando lugar a una secuencia de proporciones de respues- 
tas positivas: $\pi=\left(\pi_{1}, \pi_{2}, \pi_{3}, \ldots, \pi k\right)$, donde $\pi_{1}$ corresponde al precio de salida más bajo A1, 2 al siguiente precio de salida más bajo y así sucesivamente. El teorema de Ayer et al. (1955) muestra que si $\pi_{i}$ forma una secuencia monótona no creciente de proporciones, entonces, dicha secuencia proporciona un estimador de máxima verosimilitud de la probabilidad de aceptar el precio propuesto. Para muestras suficientemente amplias se espera que la proporción de respuestas "sí" sea estrictamente decreciente con el precio ofrecido.

Para estimar la media y la mediana, en primer lugar, se debe asumir que la interpolación lineal es una aproximación de comportamiento adecuada entre los cinco puntos conocidos. En segundo lugar, se debe asumir también, que $\pi=1$ cuando $A=0$ y que $\pi=0$ cuando $A=A^{*}$, es decir que, si el precio ofrecido es cero, entonces la probabilidad de aceptar el pago es la unidad y si el precio es $A^{*}$ entonces es cero, ya que se supone que es demasiado alto y, en consecuencia, nadie acepta el pago propuesto. Por tanto, se asume implícitamente un punto de truncamiento $A^{*}$ de tal forma que la probabilidad de obtener una respuesta positiva es cero (Salazar \& García, p. 12).

Puesto que no es posible renunciar al uso del bien (Suministro de agua), al menos, mientras no hayan otras opciones, es preferible usar la media en lugar de la mediana, de esta manera se obtendrá la suma de dinero que el individuo estaría dispuesto a pagar para que se produzca la mejora. Además, esta medida de bienestar es el valor esperado de $C$, denominado $C+$. Se calcula con el método de integración por partes, a partir de la función de probabilidad acumulada (Hanemann, 1989; Ardila, 1993).

Para ello, se usa la forma funcional más sencilla que es la lineal de Hanemann (1984), $\Delta V=a-\beta X+\eta$. Para tener mejor análisis de las medidas de bienestar es aceptable adaptar la forma funcional lineal de Hanemann agregándole otras variables (A) que resultan estadísticamente significativas, obteniendo así la siguiente expresión $\Delta V=\alpha-\beta 1 X+\beta 2 A+\eta$.

Para la forma funcional lineal de la función de utilidad indirecta $\Delta v=\alpha_{j}+\beta y+\varepsilon_{j}, \Delta v$ queda expresado como queda expresado como:

$$
\begin{gathered}
\Delta v=\left(a_{1}-a_{o}\right)-\beta C+\varepsilon_{1}-\varepsilon_{o} \\
\alpha-\eta=\beta C
\end{gathered}
$$

Despejando C: $C=\frac{\alpha-\eta}{\beta}$

De aquí se derivan las medidas de cambio en el bienestar, que serán, para este caso:

$$
\begin{gathered}
\text { Media } E(C)=\alpha / \beta \\
\text { Mediana } E\left(C^{*}\right)=\alpha / \beta
\end{gathered}
$$

El operador esperanza, es definido por Hanemann (1984) como la función generadora de momentos de $v$, la cual asume la forma:

$$
E\left(e^{\eta / \beta}\right)=\frac{\pi}{\beta * \operatorname{sen}(\pi / \beta)} \text { para el caso Logit y } E\left(e^{\eta / \beta}\right)=\exp \left(\frac{1}{2 \beta^{2}}\right) \text { para el caso probit. }
$$




\section{Descripción del área de estudio}

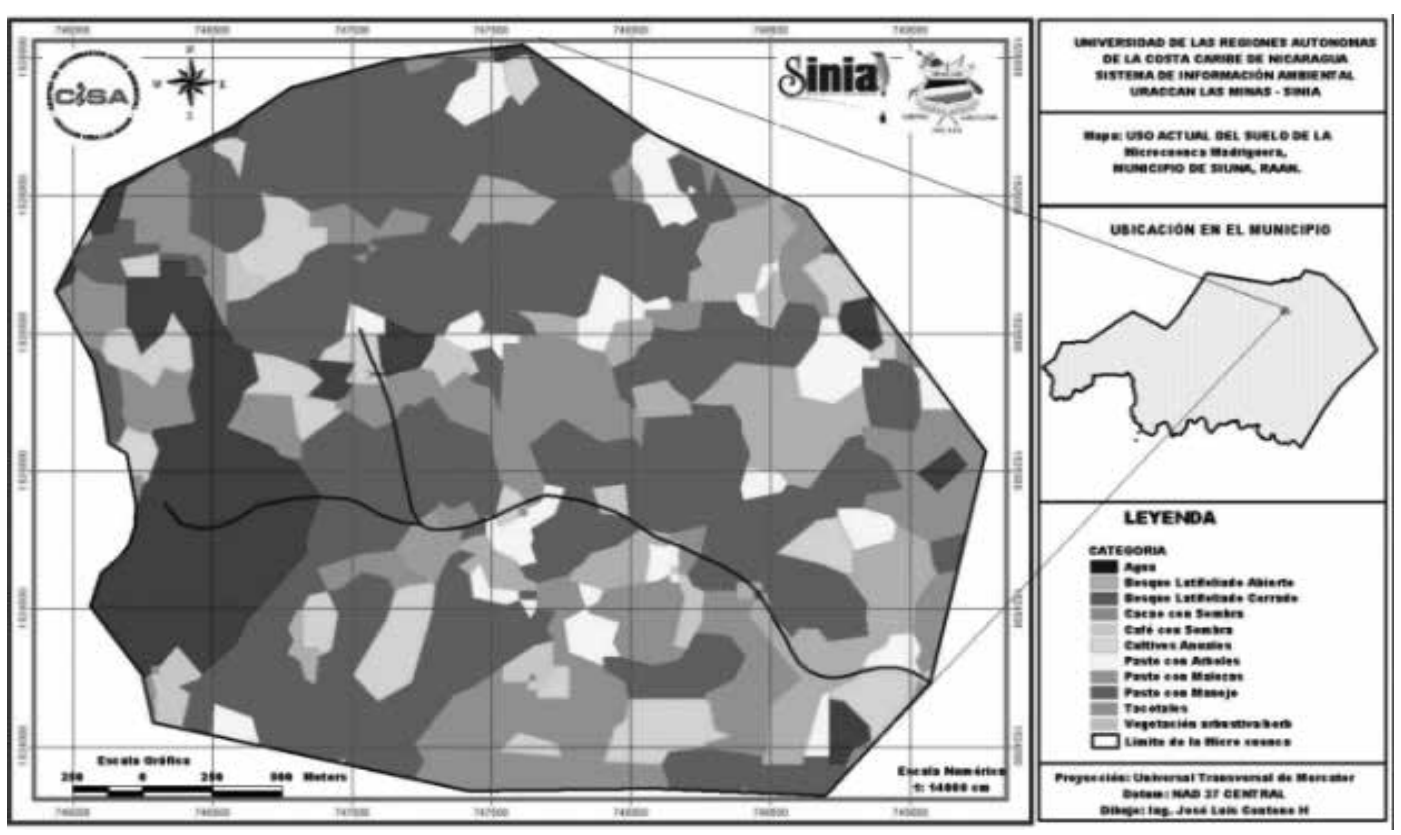

La microcuenca Madriguera, está ubicada en la parte noreste del área urbana de Siuna a unos $15 \mathrm{Km}$ de distancia con una altitud promedio de $500 \mathrm{msnm}^{2}$. En dicha área existen unas 92 hectáreas de bosque latifoliado denso, con alta diversidad florística, faunística y paisajística, propia de los bosques tropicales húmedos, con funciones características como regulador del flujo hidrológico, creando condiciones para la formación de suelo, control de procesos erosivos, el ciclo de nutrientes, producción de alimentos, especialmente para la vida silvestre, refugio de animales silvestres, producción de recursos genéticos, otros. Los pobladores se benefician directa o indirectamente de las interacciones ecológicos de esta área.

De esta área emanan afluentes de gran calidad hídrica, cuyas aguas son conducidas por tuberías hacia una población urbana con la cual resuelven el problema de escases de agua en la zona urbana. Actualmente el bosque presenta la tendencia a ser convertido en áreas agrícolas, y con ello, se disminuirá el potencial del mismo, como productor y regulador hídrico, con ello también se reduce la posibilidad de que la población urbana continúe recibiendo el beneficio (agua) generado por este bosque.

El objetivo es poner de manifiesto alternativas económicas que reporten ganancias a la sociedad en función de los recursos naturales y el ambiente de manera sostenible y sustentable, a través de la valoración del Servicio Ambiental Hídrico generado del bosque latifoliado de la microcuenca Madriguera, a través de la valoración de los individuos sobre un componente o cualidad del ambiente, como expresión directa de éstos, Disposición a pagar (DAP) familias de Siuna o Disposición a aceptar (DAA) familias residentes en el área proveedora del servicio, mediante encuestas.

Es evidente que se tienen dos poblaciones, primera, las cincuenta familias residentes en el área propiamente de la microcuenca Madriguera, que dependen de la agricultura de subsistencia y de la materia prima generada por la existencia del bosque, por tanto, son de escasos recursos económicos, estas son las llamadas a ser compensadas (50), por los beneficios que genera el bosque y por retomar acciones que permitan otras alternativas de aprovechamiento, inversiones y manejo forestal. La segunda, las familias residentes en el área urbaun proyecto conservacionista que asegure la continuidad de los servicios ambientales provenientes de ella y, quienes serían requeridos a pagar si se implementara. Así como su

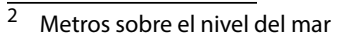


localización geográfica. Una tercera sección, que incluyó información (socioeconómica) sobre la persona encuestada.

\section{Encuesta piloto}

Se realizó una encuesta piloto con formato abierto en familias residentes en el área urbana de Siuna (no circundantes). La muestra piloto fue de 25 personas encuestadas para determinar la distribución de la Disposición a Pagar (DAP) y evaluar el nivel de entendimiento de las preguntas planteadas en la encuesta, así como el escenario de valoración y eficiencia de la ayuda visual (Mapas de cambio de uso del suelo 2005 y 2010).

\section{Cálculo del tamaño de las muestras}

El Universo consistió en un total de 2,342 viviendas de las cuales 2,070 están ocupadas según el censo de Instituto Nacional de Información de Desarrollo (INIDE, 2005, p. 41). Sin embargo, datos estadísticos incorporados en el Diagnóstico de agua y saneamiento del municipio de Siuna, presentado en el año 2011 en el área urbana, solo 1,741 viviendas (45\%), tiene el servicio de agua distribuido por tubería, a través de la Empresa Municipal de Agua Potable (EMAPSA). De acuerdo con el INIDE (2012), 1,054 viviendas no tienen acceso a este servicio y según estadísticas nacionales, en promedio una familia representativa nicaragüense está conformada por 506 integrantes.

El sistema de agua de Uly sirve a 1,273 viviendas, lo cual corresponde al $73 \%$ del total de viviendas que atiende la Empresa Municipal de Agua Potable S.A. (EMAPSA). Este sistema cuenta con tratamiento por lo cual el agua es apta para el consumo humano. El 37\% restante (644 viviendas), recibe agua cruda (sin tratamiento) a través de la red antigua de la Microcuenca Madriguera. En ésta última, se trabajó con la población demandante del recurso hídrico de esta microcuenca, es decir, las 644 viviendas. Los encuestados fueron personas mayores de 16 años, $y$ preferiblemente, jefes de hogar, representativas de la población en estudio, se aplicó al azar, una cantidad de 246 encuestas a finales del mes de enero.

La fórmula de cálculo del tamaño de las muestras conociendo el tamaño de la población fue la siguiente (Spiegel, 1988):

Donde:

\begin{tabular}{|l|l|}
\hline $\mathrm{N}=$ Tamaño de la población (hogares) & $\begin{array}{l}\text { E: Error máximo admisible del } \\
10 \%\end{array}$ \\
$\mathrm{Z}=1,96$ (nivel de significancia del $5 \%)$ & $\mathrm{n}$ : El tamaño de la muestra fue \\
$\mathrm{p}, \mathrm{q}=$ variabilidad (se asumió que es del $50 \%)$ & 241 viviendas. \\
\hline
\end{tabular}

\section{Técnica de muestreo aleatorio simple}

Se utilizó el muestreo aleatorio simple, cuyo diseño en campo trató de abarcar representatividad numérica y espacial dentro del marco muestral definido y se trabajó en función de las viviendas, utilizando vectores de pagos diferentes en viviendas de manera alterna.

\section{Vehículo del vector de pago}

Un criterio muy importante para el método de pago es que este sea creíble. Se recomendó como vehículo la imposición de contribuciones fijas mensuales, a través del incremento en la tarifa de agua durante un año.

\section{Resultados}

Determinación de la distribución estadística de la disponibilidad a pagar (DAP) a partir de la pre-encuesta

La pre-encuesta fue útil para determinar la distribución de la DAP y evaluar el nivel de entendimiento de las preguntas planteadas en la encuesta, así como el escenario de valoración. Luego de aplicar la pre-encuesta con formato abierto, se utilizó el test Kolmogorov-Smirnov (K-S) para una muestra para determinar la distribución de la DAP. Por tanto, a través del análisis del test $(p<\alpha=0.05)$ se determinó que la DAP tiene una distribución asimétrica log - normal.

Tabla 1. Proporción de respuestas positivas a las cantidades de pagos ofrecidas

\begin{tabular}{|l|l|l|l|l|}
\hline \multicolumn{1}{|c|}{ BID } & ANS $(+)$ & ANS $(-)$ & ANSTOTAL & Prop. ANS $(+)$ \\
\hline 30 & 49 & 0 & 49 & 1.0 \\
\hline 50 & 45 & 4 & 49 & 0.92 \\
\hline 70 & 41 & 7 & 48 & 0.85 \\
\hline 100 & 38 & 12 & 50 & 0.76 \\
\hline 200 & 34 & 16 & 50 & 0.68 \\
\hline
\end{tabular}

Fuente: elaboración propia. 
ANS $(+)=$ Respuestas positivas obtenidas ante el ofrecimiento de las cantidades (BID) del vector de pagos.

ANS $(-)=$ Respuestas negativas obtenidas ante el ofrecimiento de las cantidades (BID) del vector de pagos.

ANSTOTAL $=$ Respuestas totales.

Prop.ANS (+) = Proporción de respuestas positivas.

La tabla 1 muestra que, a mayor BID ofertado, menos disposición a pagar, y viceversa, generalmente, los que están dispuesto a pagar un BID más alto, están relacionado con mayores ingresos familiares; pero, también con la escases o carencia del suministro del recurso hídrico en sus hogares, es decir, los que no tienen distribución de agua en sus viviendas tienen mayor disposición por un BID mayor.

\section{Características socio económicas}

De las personas encuestadas (246) en el área urbana de Siuna, el $53.7 \%$ son mujeres y $46.3 \%$, hombres, una proporción muy representativa de ambos sexos. En cuanto a la disposición a pagar, existe similitud en los resultados (51.7\%, mujeres), pero en cuanto a la negativa a pagar, la proporción de mujeres es casi el doble a la de los hombres, $64.1 \%$ y $35.9 \%$, respectivamente. Lo que indica que la muestra contiene información bien proporcionada respecto de ambos sexos.

Tabla 2. Principales características socio económicas, área urbana, Siuna

\begin{tabular}{|l|l|l|l|l|}
\hline \multicolumn{1}{|c|}{ Características } & Mínimo & \multicolumn{1}{c|}{ Máximo } & \multicolumn{1}{c|}{ Media } & \multicolumn{1}{c|}{ Desviación típica } \\
\hline Ingresos (C\$) & 1000 & 5700 & 3808.23 & 1329.476 \\
\hline Nivel de Educación (Años) & 1 & 20 & 9.01 & 5.089 \\
\hline Tamaño de la familia (Número) & 1 & 9 & 4.47 & 1.603 \\
\hline Edad (años) & 15 & 77 & 36.68 & 10.799 \\
\hline & & & & \\
\hline
\end{tabular}

Las principales actividades económicas, son pequeños negocios yasalariados del Ministerio de Educación y el Ministerio de Salud, esto indica que se trata de una población de escasos recursos económicos.

\section{Percepción acerca de la microcuenca Madriguera}

Tabla 3. Respuestas a la percepción acerca de la micro cuenca Madriguera

\begin{tabular}{|l|l|l|l|}
\hline \multicolumn{1}{|c|}{ Aspectos } & \multicolumn{1}{|c|}{ Si (\%) } & \multicolumn{1}{|c|}{ No (\%) } & Total (\%) \\
\hline Conozco la micro cuenca madriguera & 74.0 & 26.0 & 100 \\
\hline Sé dónde viene el agua que consumo & 95.9 & 4.1 & 100 \\
\hline Sé qué es un servicio ambiental & 66.8 & 33.2 & 100 \\
\hline La microcuenca está bajo amenaza & 96.4 & 3.6 & 100 \\
\hline Debe brindársele protección a la microcuenca & 99.5 & 0.5 & 100 \\
\hline Pagaría & 88.3 & 11.7 & 100 \\
\hline
\end{tabular}

De acuerdo con los resultados, la mayoría de las personas están enteradas de la situación amenazante por la que atraviesa la microcuenca actualmente y, están conscientes de que hay que hacer algo bueno y pronto para protegerla y asegurar así, la continuidad de los servicios ambientales que brinda actualmente, esta microcuenca a una parte de la población urbana de Siuna 


\section{Principales razones y tipo de valores económicos por las que pagaría el BID ofertado}

Según la Tabla 3, el 88.3\% aceptaron pagar el BID ofrecido, a éstos se les entregó una lista de posibles razones por las que pagaría. Esta lista incluía los diferentes componentes del valor económico total, cada una con una escala de importancia del 1 al 5, realizando la siguiente pregunta: “¿me podría decir usted entre todas estas posibles razones, el orden de importancia para cada una de ellas, por lo que usted está dispuesto a pagar el monto ofrecido, por el cambio propuesto?", donde la escala 5 significa principal razón y la 1 significa mínima razón.

Tabla 4. Razones y tipos de valores económicos por las que pagaría el BID ofertado

\begin{tabular}{|c|c|c|c|c|c|c|}
\hline \multirow{3}{*}{ Razones y Tipo de valor } & \multicolumn{5}{|c|}{ Escala } & \multirow{3}{*}{ Tota } \\
\hline & \multicolumn{2}{|c|}{ Mínima } & \multicolumn{3}{|c|}{ Máxima razón } & \\
\hline & 1 & 2 & 3 & 4 & 5 & \\
\hline $\begin{array}{l}\text { Porque el bosque que aún existe en la microcuenca Madriguera protege importantes fuentes de } \\
\text { agua y regula el clima local (uso indirecto) }\end{array}$ & & 1 & 14 & 24 & 114 & 153 \\
\hline Por la biodiversidad que alberga (existencia, no uso) & 1 & 15 & 38 & 27 & 10 & 91 \\
\hline $\begin{array}{l}\text { Puedo querer visitar la Micro cuenca Madriguera y disfrutar su belleza paisajística en algún mo- } \\
\text { mento en el futuro (opción, uso) }\end{array}$ & & 59 & 17 & 10 & 2 & 88 \\
\hline Debemos proteger estas áreas para futuras generaciones (herencia, no uso) & & 7 & 26 & 41 & 64 & 138 \\
\hline $\begin{array}{l}\text { Cuando el mercado de carbono funcione y el Estado gane dinero con el Bosque existente en la } \\
\text { Microcuenca Madriguera y lo invierta en los Nicaragüenses, yo recibiría un beneficio personal por } \\
\text { pagar (directo) }\end{array}$ & 86 & 3 & & 1 & 2 & 92 \\
\hline
\end{tabular}

Al observar la Tabla 4 y apreciar la importancia relativa que tienen los diferentes tipos de servicios globales que brinda la microcuenca Madriguera, se observa que existe principalmente una clara preocupación por la pérdida de su capacidad para proteger y conservar el recurso hídrico y su implicancia en el suministro de este servicio a la población urbana de Siuna, (Uso Indirecto) y la idea de que generaciones futuras no puedan disfrutar de los beneficios de este ecosistema (Herencia), le sigue, la preocupación de que desaparezcan especies y se pierda biodiversidad (Existencia), en últimos ordenes de importancia, están la preocupación de perder la posibilidad de poder visitarla en algún momento en el futuro para disfrutar de su belleza paisajística y, conservarlo para no perder un beneficio personal como los incentivos por la captura de carbono (opción y uso directo).

\section{Principales razones por las que no pagaría el BID propuesto}

En los resultados de la Tabla 5 se observa que la negativa a aceptar el pago propuesto obedece, principalmente, a una restricción presupuestaria. Manifiestan mayoritariamente que no aceptan pagar porque: "No tienen dinero suficiente" (69.6\%), seguido de cierto nivel de incertidumbre de los encuestados "No estoy de acuerdo con el programa" (8.7\%), y "Creo que el fondo será mal utilizado" (8.7\%).

Tabla 5. Razones por las que no pagaría el BID propuesto

\begin{tabular}{|l|l|l|}
\hline \multicolumn{1}{|c|}{ Motivo } & \multicolumn{1}{|c|}{ Número } & \multicolumn{1}{c|}{ Porcentaje } \\
\hline No tengo los medios económicos suficientes para pagar & 16 & 69.6 \\
\hline No estoy de acuerdo con el programa. & 2 & 8.7 \\
\hline Creo que el fondo será mal utilizado. & 2 & 8.7 \\
\hline No creo que el proyecto se realice. & 1 & 4.3 \\
\hline La corrupción puede evitar que los recursos lleguen a su destino. & 1 & 4.3 \\
\hline Es la municipalidad la que debe hacerse cargo. & 1 & 4.3 \\
\hline Total de respuestas & 23 & 100 \\
\hline
\end{tabular}




\section{Descomposición porcentual de la DAP}

Para poder descomponer la DAP se realizó la siguiente pregunta, de la cantidad de córdobas (BID ofrecido), que usted está dispuesto a pagar para la conservación de parte de la microcuenca Madriguera “¿podría decirme como distribuye en forma porcentual según su orden de importancia esa cantidad entre las razones por la que usted pagaría?, Usted puede asignar o\% a una opción y distribuir el 100\% entre las demás opciones según su preferencia o asignar 100\% a una sola opción".

La desagregación porcentual de la DAP de cada individuo, permitieron obtener los valores promedios globales para cada componente del valor económico total. Se encontró que el valor de uso indirecto fue el más alto, (77.6 \%), seguido por los valores de Herencia, Directo, Existencia y Opción de uso con 74.4\%, $46.9 \%, 46.4 \%$, y $44.9 \%$, respectivamente.

Tabla 6. Descomposición porcentual de la DAP

\begin{tabular}{|l|l|l|}
\hline \multicolumn{1}{|c|}{ Tipo de valor económico } & \multicolumn{1}{|c|}{ Razón } & Valores (\%) DAP desagregada \\
\hline Uso indirecto & $\begin{array}{l}\text { Porque el bosque que aún existe en la Microcuenca Madriguera prote- } \\
\text { ge importantes fuentes de agua y regula el clima local. }\end{array}$ & 77.6 \\
\hline Herencia, no uso & Debemos proteger estas áreas para futuras generaciones. & 70.4 \\
\hline Uso directo & $\begin{array}{l}\text { Cuando el mercado de carbono funcione y el Estado gane dinero con } \\
\text { el Bosque existente en la Microcuenca Madriguera y lo invierta en los } \\
\text { nicaragüenses, yo recibiría un beneficio personal por pagar. }\end{array}$ & 46.9 \\
\hline Existencia, no uso & Por la biodiversidad que alberga. & 46.4 \\
\hline Opción, uso & $\begin{array}{l}\text { Puedo querer visitar la Micro cuenca Madriguera y disfrutar su belleza } \\
\text { paisajística en algún momento en el futuro. }\end{array}$ & 44.9 \\
\hline
\end{tabular}

Estos valores guardan relación con los encontrados en la tabla 4, en el sentido que siguen el mismo orden en el nivel de importancia asignado para cada componente del valor económico total del bosque de la Micro cuenca, es decir el orden de importancia es: Valor de Uso indirecto, Valor de Herencia, Valor de Uso directo Valor de Existencia y Valor de Opción.

\section{Estimaciones paramétricas y su forma funcional lineal}

Tabla 7. Coeficientes de la estimación paramétrica y las pruebas t

\begin{tabular}{|c|c|c|}
\hline \multirow{3}{*}{ Variable } & \multicolumn{2}{|c|}{$\begin{array}{c}\text { Forma funcional lineal } \\
\text { Hanemann (1984) } \Delta V=\alpha-\beta X\end{array}$} \\
\hline & \multicolumn{2}{|c|}{ Distribución } \\
\hline & Probit & Logit \\
\hline Constante & $4.51041739(0.000)$ & $23.4840953(0.000)$ \\
\hline $\mathrm{BID}(\mathrm{X})$ & $-0.00876561(-4.354)$ & $-0.01572370(-4.299)$ \\
\hline R2 McFadden & 0.27323 & 0.36036 \\
\hline Akaike & 0.74123 & 0.59525 \\
\hline Schwarz & 227.91235 & 227.97336 \\
\hline Chi cuadrado & 58.77669 & 58.71567 \\
\hline$\%$ Pred. Corr. & $86.179 \%$ & $90.306 \%$ \\
\hline Log likelihood funtion & -78.17152 & -78.20203 \\
\hline
\end{tabular}

Nota: Datos estimados sobre la base de cinco vectores de pagos (30, 50, 70, 100 y 200).

Para la estimación de las medidas de bienestar media y mediana, se utilizó el programa econométrico profesional LIMDEP, versión N LOGIT 3.o. En las regresiones, la variable dependiente denominada ANS, está representada por las respuestas si/no de disposición a pagar declaradas por los individuos. Por otro lado, las variables independientes, utilizadas en las distribuciones Logit y probit, fueron: pago ofertado 
(BID), ingreso (INGRESO), nivel de estudio (NIVED), tamaño de la familia (NROPER), horas (HORAS), sabe (SABE), protección (PROTEC), servicio ambiental (SSAMB), bosque amenazado (BOSQUEAM), estado civil (ECIVIL) edad (EDAD) de los entrevistados y sexo (SEXO).

Las variables estadísticamente significativas al $5 \%$, para ambos modelos lineales fueron; pago ofertado (BID), ingreso nivel de educación y número de personas en la familia. Sin embargo, en este estudio se considera como única variable explicativa el vector de pagos (BID) (Ver Tablas 7 y 8), esto con el fin de poder comparar las dos distribuciones logit y probit dentro de las estimaciones paramétricas bajo el mismo set de variables explicativas.

$\mathrm{Al}$ analizar las variables significativas, la variable BID negativa se hace consistente con la teoría económica, a mayor cantidad de pago ofertado, existe menor DAP por parte de los encuestados. En relación a la bondad de ajuste de las distribuciones de modelo lineal, analizando el indicador R McFadden y Akaike (Véase Tabla 7), indica, al parecer, que la distribución Logit presenta un mejor ajuste; el porcentaje de predicciones correctas corrobora esta apreciación, es decir, la cantidad de aciertos al predecir el comportamiento de la variable dicotómica de respuesta (ANS) coincide con la distribución Logit. Sin embargo, Schwarz y el test chi cuadrado indican un mejor ajuste, sutil, para la distribución Probit.

Tabla 8. Coeficientes de la estimación paramétrica y las pruebas $t$

\begin{tabular}{|l|l|l|}
\hline \multirow{2}{*}{ Variable } & \multicolumn{2}{|c|}{ Datos descriptivos. 30, 50, 70 y 100} \\
\cline { 2 - 3 } & \multicolumn{2}{|c|}{$\begin{array}{c}\text { Forma funcional lineal } \\
\text { Hanemann }(1984) \Delta V=a-\beta X\end{array}$} \\
\cline { 2 - 3 } & \multicolumn{2}{|c|}{ Distribución } \\
\cline { 2 - 3 } & \multicolumn{2}{|c|}{ Probit } \\
\hline Constante & $10.8113850(0.000)$ & $52.5133272(0.000)$ \\
\hline BID $(X)$ & $-0.02937266(-3.824)^{*}$ & $-0.05762271(-3.829)^{*}$ \\
\hline R2 McFadden & 0.35527 & 0.36036 \\
\hline Akaike & 0.59893 & 0.59525 \\
\hline Schwarz & 160.00619 & 159.28446 \\
\hline Chi cuadrado & 50.35862 & 51.08036 \\
\hline \% Pred. Corr. & $89.796 \%$ & $90.306 \%$ \\
\hline Log likelihood funtion & -45.69535 & -45.33448 \\
\hline
\end{tabular}

*= Significativo al $5 \%$.

En la tabla 8 se muestran los resultados de los datos con un vector de pago menos, el de 200 córdobas, para obtener una estimación más conservadora.

Las variables estadísticamente significativas al 5\%, tanto para el modelo lineal Probit como para el modelo lineal Logit son: BID, ingreso, Nivel de educación, número de personas en la familia y la edad.

$\mathrm{Al}$ analizar las variables significativas, la variable BID presenta signo negativo, lo cual la hace consistente con la teoría económica, porque a mayor cantidad de pago ofertado, existe menor DAP por parte de los encuestados. En relación a la bondad de ajuste de las distribuciones de modelo lineal, analizando el indicador R McFadden, Akaike y Schwarz (Véase Tabla 7), indican que al parecer, la distribución Logit presenta un mejor ajuste; el test chi cuadrado, el porcentaje de predicciones correctas corrobora esta apreciación, es decir, la cantidad de aciertos al predecir el comportamiento de la variable dicotómica de respuesta (ANS) coincide con la distribución Logit. 
Tabla 9. Medidas de cambio en el bienestar, estimaciones paramétricas, Siuna.

\begin{tabular}{|l|l|l|}
\hline \multirow{2}{*}{$\begin{array}{c}\text { Medida de cambio en el bienestar } \\
\text { Paramétrica }\end{array}$} & \multicolumn{2}{|c|}{$\begin{array}{c}\text { Forma funcional Lineal Hanemann (1984) } \\
\Delta V=\mathbf{a}-\beta A_{\mathbf{i}}\end{array}$} \\
\cline { 2 - 3 } & \multicolumn{1}{|c|}{ Probit C\$/mes } & \multicolumn{1}{c|}{ Logit $\mathbf{C} / \mathrm{mes}$} \\
\hline Media = Mediana * & $219.70[162.36-277.03]$ & $218.62[157.24-279.99]$ \\
\hline
\end{tabular}

Analizando los resultados de forma puntual, bajo el enfoque de estimación paramétrica. La media que coincide con la mediana para el caso de la forma funcional lineal, se obtuvieron valores de $C \$ 219.70$ y $C \$$ 218.62 mensual, para la distribución probit y logit, respectivamente, con cinco vectores de pagos 30, 50, 70, 100 y 200. En ambos casos, cuando la media y la mediana, se estiman a partir de los ingresos únicamente, los valores son ligeramente superior a los estimados cuando se adicionan otras variables ambientales y socioeconómicas.

\begin{tabular}{|l|c|c|}
\hline \multirow{2}{*}{$\begin{array}{c}\text { Medida de cambio en el } \\
\text { bienestar Paramétrica }\end{array}$} & \multicolumn{2}{|c|}{$\begin{array}{c}\text { Forma funcional Lineal Hanemann (1984) } \\
\Delta V=\mathbf{a}-\beta A_{\mathbf{i}}\end{array}$} \\
\cline { 2 - 3 } & \multicolumn{1}{|c|}{ Probit C\$/mes } & \multicolumn{1}{c|}{ Logit C\$/mes } \\
\hline Media = Mediana * & $116.29[94.83-137.75]$ & $114.41[92.38-136.44]$ \\
\hline
\end{tabular}

La media que también coincide con la mediana para el caso de la forma funcional lineal, se obtuvieron valores de $C \$ 116.29$ y $C \$ 114.41$ mensual para la distribución probit y logit, respectivamente, con cuatro vectores de pagos, 30, 50, 70 y 100.

\section{Agregación de los beneficios}

Si se desean agregar los resultados obtenidos se deben recordarse los datos poblacionales que se tienen según estadísticas nacionales, así, se tienen, 644 viviendas en el área urbana de Siuna. Si se multiplica, el valor puntual de la medida de cambio en el bienestar, en este caso el valor de la media o mediana (el menos conservador) se obtiene un valor de es $219.70 \mathrm{C} \$ / \mathrm{mes}$, equivalente a $9.05 \$ \mathrm{US} / \mathrm{mes}$-tipo de cambio promedio mes de febrero de 2013, $1 \$ U S=24,28 C \$$ (Banco Central de Nicaragua, 2013).

De otra forma, si se consideran intervalos para el valor elegido, el rango estará entre 162.36 y $277.03 C \$ /$ mes equivalente a 6.69 y $11.41 \$$ US $/$ mes. Asumiendo el valor más conservador ( $C \$ 114.41 / \mathrm{mes})$, multiplicado por el número de viviendas es, $C \$ 73,680.04$ mensual, $C \$ 884,160.48$ al año $(36,415.18$ US/año). Si se consideran intervalos para el valor elegido, el rango estará entre 92.38 y $136.44 C \$ /$ mes equivalente a 3.80 y $5.60 \$ U S / m e s$.

Los valores obtenidos en esta estimación son relativamente altos, sin embargo, estos pobladores reciben el suministro de agua cada ocho días y en tiempo promedio de ocho a doce horas. Actualmente una minoría paga el mínimo 50 córdobas mensuales, y no existen medidores por lo que no existe control del suministro y gasto de agua por viviendas.

\section{Conclusiones}

La microcuenca Madriguera es un área importante que genera servicios ambientales para los pobladores urbanos y rurales del municipio de Siuna en las veredas objeto del área de estudio y se encuentra en amenazas por actividades económicas.

Tres de cada cuatro encuestados, dijeron conocer el área de microcuenca, al menos 9 de cada 10, saben de donde proviene el agua. 
Con respecto a la DAP, el $84 \%$ de los entrevistados respondió afirmativamente y a medida que los montos contenidos (BID) aumenta, la probabilidad de obtener respuestas positivas disminuye. Los que participan expresan que el pago lo hacen atendiendo al tipo de valor económico de uso indirecto (77.6\%) y al de herencia, no uso (70.4\%). Los valores de uso directo, existencia y de opción, son ligeramente duplicados por los anteriores.

Las características socioeconómicas más importantes, son el ingreso $(C \$)(p<0.01)$, el nivel de educación (años) ( $\mathrm{p}<0.05)$ y el número de personas en la familia $(\mathrm{p}<0.05)$.

La edad, sexo, conocimiento sobre servicio ambiental, el estado civil y otras variables inherentes al problema no tienen peso en la respuesta positiva de DAP.

El beneficio agregado, para el valor más conservador como medida de cambio de bienestar, es $C \$ 116.29$. Ello implica, $C \$ 74890.76$ mensuales, unos $C \$ 898689.12$ al año.

Teniendo en cuenta que los beneficiarios (pobladores urbanos) de los servicios ambientales valoran su provisión. Resta aplicar herramientas adicionales para indagar y decidir el mecanismo idóneo para las condiciones biofísicas y socioeconómicas de la zona.

\section{Lista de referencias}

Almansa, C., \& Calatrava, J. (Mayo de 2006). Valoración contigente del control de la erosión. Pamplona, España.

Aviléz, G., Huato, L., Troyo-Diéguez, E., Murillo, B., García, J., \& Beltran, L. (2010). Valoración económica del servicio hidrológico del acuífero de La Paz, b.c.s.: Una valoración contingente del agua de uso municipal. Frontera norte, 22 (34), 103-128.

Barzev, R. (2002). Guía metodológica valoración económica de bienes, servicios e impactos ambientales. Serie técnica 04. Managua, Nicaragua: Corredor biologico mesoamericano.
Cerda, A. (9 de Julio de 2003). Valoración económica del Medio Ambiente. Cartagena de Indias, Colombia.

Cerda, A., García, L., Bahamondez, A., \& Poblete, V. (2010). Disposición a pagar para mejorar la calidad del aire en Talca, Chile: comparación entre usuarios y no usuarios de chimeneas a leña. Lecturas de Economía (72), 195-211.

Cerda, A., Rojas, J., \& García, L. (2007). Disposición a pagar por un mejoramiento en la calidad ambiental en el Gran Santiago, Chile. Lecturas de Eonomía , 143-16o.

Hanemann, M. (2012). Valuando el medio ambiente a través de la valoración contingente. Gaceta de Economía (16), 18-46.

INIDE. (Junio de 2005). El Instituto Nacional de Información de Desarrollo (INIDE). Recuperado el Febrero de 2013, de Cifras Municipales: http://www.inide.gob.ni/censos2005/ CifrasMun/RAAN/SIUNA.pdf

Larach, M. A. (7 de Noviembre de 2000). Comercio $y$ Medio Ambiente en la OMC. Recuperado el o5 de Enero de 2013, de Internalización de los costos ambientales: http://www.eclac.cl/ publicaciones/xml/6/4216/internal.htm

Matínez, M., \& Dimas, L. (Julio de 2011). Valoración Económica de los servicios hidrologicos: Sub cuenca del Río Teculután, Guatemala. Recuperado el 16 de Febrero de 2013, de http://awsassets. panda.org/downloads/valoracion_economica_rio_teculutan.pdf

Mendieta, J. C. (Enero de 200o). Economía ambiental. Recuperado el 25 de Octubre de 2012, de http://es.scribd.com/doc/58310204/16/ Variacion-Compensatoria-y-VariacionEquivalente

Riera, P. (1994). Manual de valoración contingente. Recuperado el 15 de Febrero de 2013, de manualcvm2.pdf: http://pagines.uab.cat/pere. riera/sites/pagines.uab.cat.pere.riera/files/ manualcvm2.pdf

Salazar, S. d. (10 de Mayo de 200o). Estimación de medidas de bienestar mediante valoración contingente. Una aproximación no paramétrica. 
URACCAN

Salazar, S. d., \& García, L. (s.f.). Disposición a pagar versus disposición a ser compensado por mejoras medio ambientales: evidencia empírica. Valencia, España.

Villena, M., \& Lafuente, E. (2012). Valoración económica de bienes ambientales por beneficiarios circundantes y no circundantes. Cuadernos de Economía , 31 (56), 4-72. 